\title{
Association between fetuin-A levels with insulin resistance and carotid intima-media thickness in patients with new-onset type 2 diabetes mellitus
}

\author{
LIANG YIN $^{1 *}$, WEI-JUAN CAI ${ }^{2 *}$, XIANG-YUN CHANG ${ }^{1}$, JUN LI $^{1}$, XIANG-HUI SU ${ }^{1}$, \\ LING-YUN ZHU ${ }^{1}$, XIAO-LI WANG ${ }^{1}$ and KAN SUN ${ }^{1}$
}

Departments of ${ }^{1}$ Endocrinology and Metabolism and ${ }^{2}$ Clinical Laboratory, The First Affiliated Hospital, Shihezi University School of Medicine, Shihezi, Xinjiang 832002, P.R. China

Received December 14, 2013; Accepted July 24, 2014

DOI: 10.3892/br.2014.356

\begin{abstract}
Fetuin-A, which is known to inhibit insulin signaling and pathological calcification, has emerged as a diabetes risk biomarker. In the present study, the association between the fetuin-A levels with insulin resistance (IR) and carotid intima-media thickness (CIMT) was investigated in patients with new-onset type 2 diabetes mellitus (nT2DM). A total of 100 patients with nT2DM (nT2DM group) and 100 normal glucose tolerance (NGT group) controls were evaluated. The serum fetuin-A level was measured by a commercial solid-phase ELISA kit. The estimate of IR was calculated by homeostasis model assessment (HOMA-IR). CIMT was measured by B-mode ultrasound. The association between the serum fetuin-A levels and the metabolic parameters was also analyzed. The serum fetuin-A levels were increased significantly in the nT2DM group compared to the NGT group $(368.5 \pm 15.6 \mathrm{mg} / \mathrm{ml}$ vs. $152.7 \pm 7.1 \mathrm{mg} / \mathrm{ml}, \mathrm{P}<0.01)$. Fetuin-A was positively correlated with HOMA-IR, CIMT, glycated hemoglobin, triglyceride, low-density lipoprotein cholesterol, body mass index, systolic blood pressure, fasting blood glucose and $2 \mathrm{~h}$ post-glucose load blood glucose $(\mathrm{P}<0.05$ and $\mathrm{P}<0.01$ ), but negatively correlated with fasting plasma insulin, 2 h plasma insulin after glucose overload, high-density lipoprotein cholesterol and HOMA- $\beta$-cell insulin secretion index $(\mathrm{P}<0.05$ and $\mathrm{P}<0.01)$. To the best of our knowledge, the study demonstrated for the first time that there is a significant association between the serum fetuin-A levels with IR and CIMT in nT2DM. These results indicate that serum fetuin-A
\end{abstract}

Correspondence to: Dr Kan Sun, Department of Endocrinology and Metabolism, The First Affiliated Hospital, Shihezi University School of Medicine, Bei'er Road, Shihezi, Xinjiang 832002, P.R. China E-mail: sunkan_shz@126.com

*Contributed equally

Key words: new-onset type 2 diabetes mellitus, fetuin-A, insulin resistance, carotid intima-media thickness levels can be used as independent markers in the diagnosis of macroangiopathies in nT2DM.

\section{Introduction}

Atherosclerosis (AS) is the main pathological feature of type 2 diabetic macrovascular complications and is also the leading cause of mortality in these patients. The risk of AS patients with type 2 diabetes (T2DM) is higher compared to those with normal glucose tolerance (1). Patients with new-onset type 2 diabetes mellitus (nT2DM) do not have typical clinical manifestations (2), and certain patients have almost no symptoms. High blood sugar is the only indicator of nT2DM. Therefore, early detection in high-risk groups can take effective preventive measures in reducing cardiovascular mortality in patients with T2DM.

AS mainly affects the medium-sized and large arteries of the heart, brain, kidney, peripheral artery and other large vessels. AS is characterized by plaque deposits that block the flow of blood. An early sign of lesions are increases in the intima-media thickness (IMT) of the arterial wall. A previous study showed that the carotid artery IMT (CIMT) can be as a surrogate marker of coronary artery disease in diabetes (3). Therefore, it has significance for early detection and intervention of carotid artery disease in diabetic patients and allows timely treatment for the prevention of diabetic vascular complications.

Insulin resistance (IR) is one of the key pathophysiological mechanisms of T2DM, which may contribute to the development of T2DM and T2DM-associated complications. IR is also considered to be the common cause of dyslipidemia and hypertensive diseases (4).

Fetuin-A, which is a glycoprotein secreted by the liver, has emerged as a biomarker for risk of T2DM (5-10). High circulating fetuin-A levels appear to be associated with IR (11). A previous study has shown that fetuin-A induces IR by inhibiting insulin receptor autophosphorylation (12). Additionally, enhanced insulin sensitivity was observed in mice null for the fetuin gene (13). Also, fetuin-A has been shown to inhibit ectopic calcium deposition and protect from vascular calcification (14). However, contradictory results have been reported 
regarding the role of novel biomarker fetuin-A in macroangiopathies in T2DM $(15,16)$.

Additionally, to the best of our knowledge, there is no data available for the associations of fetuin-A with IR and CIMT in nT2DM. Thus, in the present study, the serum fetuin-A levels were investigated in patients with nT2DM to analyze the association of serum fetuin-A levels with IR and CIMT in patients with nT2DM.

\section{Materials and methods}

Patients. A total of 100 patients with nT2DM (nT2DM group) and 100 normal glucose tolerance (NGT group) controls were evaluated in the study. The subjects were recruited from the Departments of Endocrinology and Metabolism of First Affiliated Hospital of Shihezi University School of Medicine(Shihezi, Xinjiang, China) between 2012 and 2013. The diagnostic criteria of T2DM was based on a $75 \mathrm{~g}$ oral glucose tolerance test (OGTT) recommended by the World Health Organization criteria (17). nT2DM was defined prospectively as $\geq 2$ post-baseline fasting blood glucose (FBG) measurements $\geq 7.0 \mathrm{mmol} / 1(126 \mathrm{mg} / \mathrm{dl})$ and $\geq 1$ post-baseline glucose $>2 \mathrm{mmol} / \mathrm{l}(36 \mathrm{mg} / \mathrm{dl})$ above baseline (18). The study was performed on 100 nT2DM patients (54 males and 46 females; mean age, $55.11 \pm 12.84$ years). The NGT group, age and body mass index (BMI) matched with nT2DM group (44 males and 56 females; mean age, $52.39 \pm 13.79$ years). The exclusion criteria comprised of patients who had renal dysfunction, severe cardiac problems, uncontrolled hypertension or type 1 diabetes. The study was approved by the Institutional Ethics Committee at the First Affiliated Hospital of Shihezi University School of Medicine and conducted in accordance with the Ethical Guidelines of the Declaration of Helsinki. Written informed consent was obtained from all the patients and healthy controls prior to commencing the study.

Blood sampling. The blood samples were drawn from the antecubital vein between 09:30-10:00 am after $>8$ h of overnight fasting at the time of OGTT. The blood tubes were centrifuged for $10 \mathrm{~min}$ at $1,500 \mathrm{x} \mathrm{g}$ after clotting for $30 \mathrm{~min}$ at room temperature. The serum samples were subsequently stored at $-80^{\circ} \mathrm{C}$ until further analysis of insulin and fetuin-A levels.

Clinical data. Gender, age, height, body weight, systolic and diastolic pressures of the patients were recorded, and the BMI, homeostatic model assessment index of IR (HOMA-IR) and HOMA- $\beta$-cell insulin secretion index (HOMA-IS) $=20 \mathrm{x}$ fasting plasma insulin (FINS)/(FBG - 3.5) (19) were calculated in all the subjects.

Measurement of serum fetuin-A levels. The serum fetuin-A levels were measured with a commercially available ELISA kit (R\&D Systems, Minneapolis, MN, USA).

Measurement of CIMT. The subjects underwent a Doppler Ultrasound system (iE33; Philips Healthcare, Andover, MA, USA) with a 3-11 MHz linear-array transducer. The methods for CIMT measurement have been described in detail previously (20). The CIMT measurement was made by the same experienced ultrasonic investigator in every case.
Table I. Characteristics of the study subjects and fetuin-A levels.

\begin{tabular}{lcc}
\hline Characteristic & nT2DM & NGT \\
\hline Gender, male/female & $54 / 46$ & $44 / 56$ \\
Age, years & $55.11 \pm 12.84$ & $52.39 \pm 13.79$ \\
WHR & $0.94 \pm 0.59$ & $0.91 \pm 0.04$ \\
BMI, kg/m ${ }^{2}$ & $25.32 \pm 5.97$ & $24.38 \pm 2.19$ \\
SBP, mmHg & $134.07 \pm 15.02$ & $137.39 \pm 11.68$ \\
DBP, mmHg & $77.00 \pm 8.96$ & $81.39 \pm 8.13$ \\
FPG, mmol/l & $8.52 \pm 3.10$ & $4.85 \pm 0.48^{\mathrm{a}}$ \\
2hOGTT, mmol/l & $11.64 \pm 4.28$ & $5.13 \pm 0.48^{\mathrm{a}}$ \\
PINS, pmol/l & $40.65 \pm 27.02$ & $69.68 \pm 28.87^{\mathrm{a}}$ \\
FINS, pmol/l & $21.12 \pm 4.52$ & $35.51 \pm 3.65^{\mathrm{a}}$ \\
TC, mmol/1 & $4.92 \pm 0.92$ & $4.43 \pm 0.94^{\mathrm{a}}$ \\
TG, mmol/l & $2.12 \pm 1.81$ & $1.39 \pm 0.83^{\mathrm{a}}$ \\
LDL-C, mmol/l & $3.00 \pm 0.83$ & $2.62 \pm 0.72^{\mathrm{a}}$ \\
HDL-C, mmol/l & $1.14 \pm 0.28$ & $1.29 \pm 0.33^{\mathrm{a}}$ \\
HOMA-IR & $1.61 \pm 0.79$ & $0.65 \pm 0.05^{\mathrm{a}}$ \\
HOMA-IS & $18.49 \pm 1.72$ & $9.93 \pm 1.76^{\mathrm{b}}$ \\
HbA1c, \% & $8.78 \pm 1.80$ & $5.20 \pm 0.05^{\mathrm{a}}$ \\
Hs-CRP, mg/l & $38.5 \pm 10.3$ & $23.8 \pm 16.5$ \\
CIMT , mm ${ }^{\mathrm{c}}$ & $0.26 \pm 0.21$ & $0.13 \pm 0.08^{\mathrm{a}}$ \\
Fetuin-A, mg/ml & $368.5 \pm 15.6$ & $152.7 \pm 7.1^{\mathrm{b}}$ \\
\hline
\end{tabular}

${ }^{\mathrm{a}} \mathrm{P}<0.001$ and ${ }^{\mathrm{b}} \mathrm{P}<0.01$ compared to the NGT group; ${ }^{\mathrm{c}}$ Data with a non-Gaussian distribution that were transformed by natural logarithmic before analysis. Data are presented as mean \pm standard deviation. nT2DM, new-onset type 2 diabetes; NGT, normal glucose tolerance; WHR, waist-to-hip ratio; BMI, body mass index; SBP, systolic blood pressure; DBP, diastolic blood pressure; FPG, fasting plasma glucose; 2hOGTT, 2 h post-glucose load blood glucose; PINS, $2 \mathrm{~h}$ plasma insulin after glucose overload; FINS, fasting plasma insulin; TC, total cholesterol; TG, triglyceride; LDL-C, low-density lipoprotein cholesterol; HDL-C, high-density lipoprotein cholesterol; HOMA-IR, HOMA-insulin resistance index; HOMA-IS, HOMA- $\beta$-cell insulin secretion index; HbAlc, glycated hemoglobin; Hs-CRP, high-sensitivity C-reactive protein; CIMT, carotid intima-media thickness.

Statistical analysis. All statistical analyses were performed using the SPSS 17.0 software (SPSS, Inc., Chicago, IL, USA). All the data were expressed as mean \pm standard deviation. The differences between the groups were assessed by Student's unpaired t-test, Mann-Whitney $U$ test or $\chi^{2}$ test as appropriate. Pearson correlation test was performed to determine the associations of serum fetuin-A levels with other study variables. Variables with a non-Gaussian distribution were logarithmically transformed prior to statistical analysis. All P-values presented are two-tailed. $\mathrm{P}<0.05$ was considered to indicate a statistically significant difference.

\section{Results}

Clinical characteristics and serum fetuin-A levels. The clinical characteristics of the two groups showed significant difference in FBG, 2 h post-glucose load blood glucose (2hOGTT), FINS, 2 h plasma insulin after glucose overload (PINS), total 

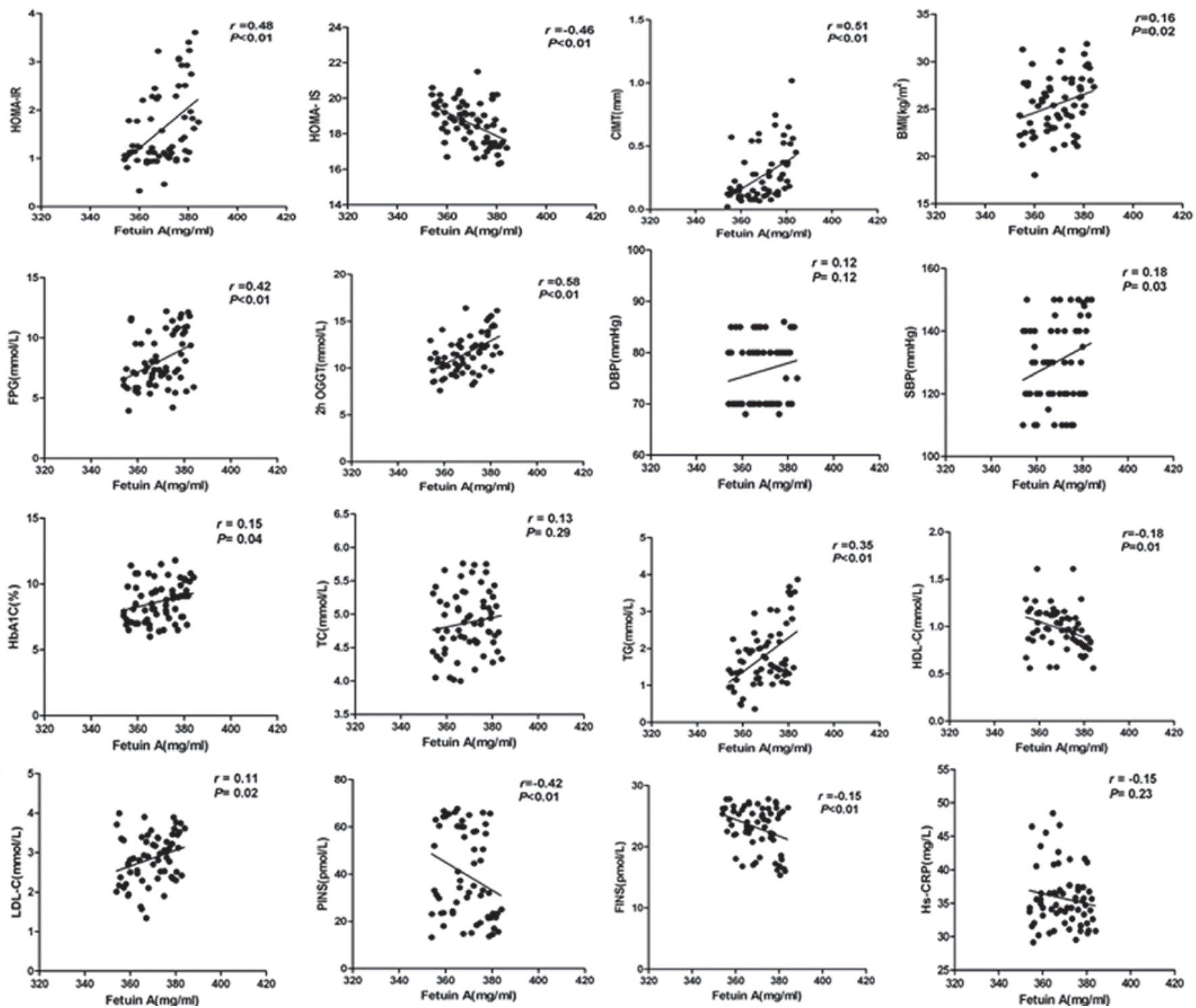

Figure 1. Correlations between the serum fetuin-A levels with the clinical parameters (Pearson correlation analysis). HOMA-IR, HOMA-insulin resistance index; HOMA-IS, HOMA- $\beta$-cell insulin secretion index; CIMT, carotid intima-media thickness; BMI, body mass index; FPG, fasting plasma glucose; 2hOGTT, $2 \mathrm{~h}$ post-glucose load blood glucose; DBP, diastolic blood pressure; SBP, systolic blood pressure; HbAlc, glycated hemoglobin; TC, total cholesterol; TG, triglyceride; HDL-C, high-density lipoprotein cholesterol; LDL-C, low-density lipoprotein cholesterol; PINS, 2 h plasma insulin after glucose overload; FINS, fasting plasma insulin; Hs-CRP, high-sensitivity C-reactive protein.

cholesterol (TC), triglyceride (TG), low-density lipoprotein cholesterol (LDL-C), high-density lipoprotein cholesterol (HDL-C), HOMA-IR, HOMA-IS, glycated hemoglobin (HbA1c) and CIMT $(\mathrm{P}<0.01$ and $\mathrm{P}<0.001$; Table 1). Gender distribution, age, waist-to-hip ratio, BMI, systolic blood pressure (SBP), diastolic blood pressure (DBP) and high-sensitivity C-reactive protein (Hs-CRP) in the nT2DM group were not statistically significant different compared to the NGT group.

Serum fetuin-A levels. There was a significant difference in the serum fetuin-A levels between the nT2DM and NGT groups (368.5 \pm 15.6 vs. $152.7 \pm 7.1 \mathrm{mg} / \mathrm{l}, \mathrm{P}<0.01$; Table I).

Correlation of serum fetuin-A levels with clinical parameters. Pearson's correlation analyses were performed to assess the associations between the serum fetuin-A levels and clinical parameters. The serum fetuin-A levels were found to correlate positively and significantly with HOMA-IR $(r=0.48, \mathrm{P}<0.01)$, CIMT ( $r=0.51, \mathrm{P}<0.01)$, HbAlc $(r=0.15, \mathrm{P}<0.05)$, SBP $(r=0.18$, $\mathrm{P}<0.05), \mathrm{LDL}-\mathrm{C}(\mathrm{r}=0.11, \mathrm{P}<0.05), \mathrm{TG}(\mathrm{r}=0.35, \mathrm{P}<0.01), \mathrm{BMI}$ $(\mathrm{r}=0.16, \mathrm{P}<0.05)$, FBG $(\mathrm{r}=0.42, \mathrm{P}<0.01)$ and 2hOGTT $(\mathrm{r}=0.58$, $\mathrm{P}<0.01)$, but negatively with FINS $(\mathrm{r}=-0.15, \mathrm{P}<0.01)$, PINS $(\mathrm{r}=-0.42, \mathrm{P}<0.01)$, HDL-C $(\mathrm{r}=-0.18, \mathrm{P}<0.05)$ and HOMA-IS $(r=-0.46, P<0.01)$, as shown in Fig. 1. However, a significant correlation was not observed between the serum fetuin-A levels and TC, DBP and Hs-CRP (Fig. 1).

\section{Discussion}

Fetuin-A is a liver-secreted, multifunctional glycoprotein (21). Previous studies have demonstrated that fetuin-A has emerged as a biomarker for risk of T2DM (5-10). In the present study, the serum fetuin-A levels were found to be elevated in patients with nT2DM compared to the NGT group. Dyslipidemia is the 
elevation of TC, TG and small dense LDL-C concentrations with low serum HDL-C levels. HDL-C has a protective role in AS, as it can remove cholesterol from cells in the artery wall (22). A previous study has demonstrated that the serum fetuin-A levels are completely associated with visceral obesity and dyslipidemia (23). In the present study, the serum fetuin-A levels were found to be positively and significantly correlated with LDL-C and TG, but negatively with HDL-C. In addition, the serum fetuin-A levels were associated with BMI, SBP, HbA1c, FBG and 2hOGTT. However, in contrast to the study, a previous study reported that fetuin-A showed a positive association with ankle brachial index and no significant correlation with the metabolic parameters (24).

In addition, it has been previously demonstrated that the serum fetuin-A level was associated with IR $(11,25,26)$. In the present study, the serum fetuin-A levels were also found to be positively and significantly correlated with HOMA-IR, but negatively with FINS, PINS and HOMA-IS. These findings indicated that fetuin-A may have a role in triggering the processes leading to IR in nT2DM patients. This result is in line with previous data from studies regarding the association between fetuin-A and IR. However, there are no associations between fetuin-A with CIMT. In the present study, the serum fetuin-A levels were found to be positively and significantly correlated with CIMT. This result indicates that the serum fetuin-A levels may be associated with macroangiopathies in nT2DM patients.

In conclusion, the present study found that serum fetuin-A levels are significantly increased and significantly associated with HbA1c, SBP, HDL-C, LDL-C, TG, BMI, FBG, 2hOGTT, FINS, PINS, HOMA-IR and HOMA-IS in nT2DM. Notably, to the best of our knowledge, this is the first time that a significant association between fetuin-A with IR and CIMT in patients with nT2DM has been shown. The findings of the study indicate that the serum fetuin-A levels can be used as independent markers in the diagnosis of macroangiopathies in nT2DM.

\section{Acknowledgements}

The present study was supported by grants from the Science and Technology Research and Development Program of Shihezi University (grant no. 2012RKXYQ-YD28) and School-level fund for the Scientific Research Project of the First Affiliated Hospital, Shihezi University School of Medicine (grant no. YL2011R018).

\section{References}

1. Pyörälä K, Laakso M and Uusitupa M: Diabetes and atherosclerosis: an epidemiologic view. Diabetes Metab Rev 3: 463-524, 1987.

2. Stumvoll M, Goldstein BJ and van Haeften TW: Type 2 diabetes: principles of pathogenesis and therapy. Lancet 365: 1333-1346, 2005.

3. Sibal L, Agarwal SC and Home PD: Carotid intima-media thickness as a surrogate marker of cardiovascular disease in diabetes. Diabetes Metab Syndr Obes 4: 23-34, 2011.

4. Reaven GM: Banting lecture 1988. Role of insulin resistance in human disease. Diabetes 37: 1595-1607, 1988.
5. Sun Q, Cornelis MC, Manson JE and Hu FB: Plasma levels of fetuin-A and hepatic enzymes and risk of type 2 diabetes in women in the U.S. Diabetes 62: 49-55, 2013.

6. Ix JH, Wassel CL, Kanaya AM, et al; Health ABC Study: Fetuin-A and incident diabetes mellitus in older persons. JAMA 300: 182-188, 2008.

7. Ix JH, Biggs ML, Mukamal KJ, et al: Association of fetuin-a with incident diabetes mellitus in community-living older adults: the cardiovascular health study. Circulation 125: 2316-2322, 2012.

8. Stefan N, Fritsche A, Weikert C, et al: Plasma fetuin-A levels and the risk of type 2 diabetes. Diabetes 57: 2762-2767, 2008.

9. Laughlin GA, Barrett-Connor E, Cummins KM, Daniels LB, Wassel CL and Ix JH: Sex-specific association of fetuin-A with type 2 diabetes in older community-dwelling adults: the Rancho Bernardo Study. Diabetes Care 36: 1994-2000, 2013.

10. Rasul S, Wagner L and Kautzky-Willer A: Fetuin-A and angiopoietins in obesity and type 2 diabetes mellitus. Endocrine 42: 496-505, 2012.

11. Song A, Xu M, Bi Y, et al: Serum fetuin-A associates with type 2 diabetes and insulin resistance in Chinese adults. PLoS One 6 : e19228, 2011.

12. Mathews ST, Chellam N, Srinivas PR, et al: Alpha2-HSG, a specific inhibitor of insulin receptor autophosphorylation, interacts with the insulin receptor. Mol Cell Endocrinol 164: 87-98, 2000.

13. Mathews ST, Singh GP, Ranalletta M, et al: Improved insulin sensitivity and resistance to weight gain in mice null for the Ahsg gene. Diabetes 51: 2450-2458, 2002

14. Schafer C, Heiss A, Schwarz A, et al: The serum protein alpha 2-Heremans-Schmid glycoprotein/fetuin-A is a systematically acting inhibitor of ectopic calcification. J Clin Invest 112: 357-366, 2003

15. Lorant DP, Grujicic M, Hoebaus C, et al: Fetuin-A levels are increased in patients with type 2 diabetes and peripheral arterial disease. Diabetes Care 34: 156-161, 2011.

16. Eraso LH, Ginwala N, Qasim AN, et al: Association of lower plasma fetuin-A levels with peripheral arterial disease in type 2 diabetes. Diabetes Care 33: 408-410, 2010.

17. Alberti KG and Zimmet PZ: Definition, diagnosis and classification of diabetes mellitus and its complications. Part 1: diagnosis and classification of diabetes mellitus, provisional report of a WHO consultation. Diabet Med 15: 539-553, 1998.

18. Freeman DJ, Norrie J, Sattar N, et al: Pravastatin and the development of diabetes mellitus: evidence for a protective treatment effect in the West of Scotland Coronary Prevention Study. Circulation 103: 357-362, 2001.

19. Matthews DR, Hosker JP, Rudenski AS, Naylor BA, Treacher DF and Turner RC: Homeostasis model assessment: insulin resistance and beta-cell function from fasting plasma glucose and insulin concentrations in man. Diabetologia 28: 412-419, 1985.

20. Aydin M, Bulur S, Alemdar R, et al; Melen Investigators: The impact of metabolic syndrome on carotid intima media thickness. Eur Rev Med Pharmacol Sci 17: 2295-2301, 2013.

21. Denecke B, Gräber S, Schäfer C, Heiss A, Wöltje M and Jahnen-Dechent W: Tissue distribution and activity testing suggest a similar but not identical function of fetuin-B and fetuin-A. Biochem J 376: 135-145, 2003.

22. Zhang Y, Zanotti I, Reilly MP, Glick JM, Rothblat GH and Rader DJ: Overexpression of apolipoprotein A-I promotes reverse transport of cholesterol from macrophages to feces in vivo. Circulation 108: 661-663, 2003.

23. Chen HY, Chiu YL, Hsu SP, et al: Association of serum fetuin A with truncal obesity and dyslipidemia in non-diabetic hemodialysis patients. Eur J Endocrinol 160: 777-783, 2009.

24. Roos M, Oikonomou D, von Eynatten M, et al: Associations of fetuin-A levels with vascular disease in type 2 diabetes patients with early diabetic nephropathy. Cardiovasc Diabetol 9: 48, 2010.

25. Stefan N,Hennige AM,StaigerH, et al: Alpha2-Heremans-Schmid glycoprotein/fetuin-A is associated with insulin resistance and fat accumulation in the liver in humans. Diabetes Care 29: 853-857, 2006.

26. Mori K, Emoto M, Yokoyama H, et al: Association of serum fetuin-A with insulin resistance in type 2 diabetic and nondiabetic subjects. Diabetes Care 29: 468, 2006. 